\title{
Impact of cigarette smoke on respiratory pathogens in biofilm production
}

\author{
Nithya Lakshmi ${ }^{1}$, Mohana Krishnan K., ," V. Darshine ${ }^{3}$, Sumathi G. ${ }^{4}$ \\ ${ }^{\mathbf{1}}$ Associate Professor, ${ }^{2}$ Professor, ${ }^{3}$ Student, ${ }^{4}$ Professor \& HOD, Dept. of Microbiology, Sri Muthukumaran Medical College \\ Hospital \& Research Institute, Chennai, Tamil Nadu, India
}

*Corresponding Author:

Email: mkprofessormicro@gmail.com

\begin{abstract}
Biofilm formation by respiratory pathogen favour their colonization, persistence and virulence. Cigarette smoke have the ability to cause induction and increase in biofilm formation. This quantitative in vitro study is aimed at understanding the dose-dependent change in the biofilm formation, which can be correlated to the degree of virulence of the respiratory pathogens.

Materials and Methods: In the present study, 77 respiratory isolates of non-smokers were included. They were processed and exposed to varying concentrations of Cigarette Smoke Extract (CSE).Quantification of biofilm production was done according to the Microtitre dish biofilm formation assay. Fischer's exact test was used to compare the biofilm production status.

Results: We observed that $42[80.77 \%$ ] isolates out of 52 biofilm producers and $12[48 \%$ ] isolates out of 25 non-biofilm producers were found to show an increase in Biofilm index (BFI) with exposure to CSE. Among the total of 77 respiratory isolates, 32 isolates accounting for $41.57 \%$ [95\% CI; 30.6\% to 52.6\%] showed a dose-related augmentation of biofilm formation on exposure to CSE. Interpretation and Conclusions: Progressive increase of biofilm with increase in concentration of CSE was the major finding. This emphasizes the need to initiate appropriate empirical therapy for smokers at the earliest.
\end{abstract}

Keywords: Biofilm producer, Cigarette smoke extract, CSE, Dose related augmentation, Respiratory pathogens, Staphylococcus aureus.

\section{Introduction}

A biofilm is a community of microbes that grow on living or inert surfaces and secrete polymers. The advantages this biofilm confers to the bacteria are: promotes sharing of the genetic material, including genes responsible for antibiotic resistance; acts as a shield against the immune system and the ability to further colonize the host upon shedding. Therefore, biofilm formation is likely to favour microbial colonization, persistence and virulence. ${ }^{1}$

A number of studies showed the ability of cigarette smoke (CS) to cause changes at the molecular level which lead to the induction and increase in biofilm formation. ${ }^{2}$ In a particular study conducted in the University of Columbia it was established that cigarette smoke rapidly altered the quorum-sensing accessory gene regulator (agr) which increased the biofilm formation via oxidative stress. ${ }^{3}$ In the study by Autunes et al, it was shown that cigarette smoke increases biofilm formation by suppressing the quorum sensing gene, rhlA and by inducing biofilm promoting Pseudomonas aeruginosa gene $(\mathrm{pilF}, \mathrm{flgK}){ }^{4}$

Though researches have shown a definite role of CS on biofilm formation, ${ }^{3,5,6}$ there are not quite a lot of studies which show the dose-dependent relationship of CS on these pathogens, as in, if the amount of cigarette smoke you are exposed to affects the degree of biofilm formation. Therefore, this quantitative in vitro study is aimed at understanding the dose-dependent change in the biofilm formation.

The association between biofilm production with persistent infection and antibiotic therapy failure is well established by various studies. ${ }^{7,8}$ Stewart et al showed that biofilms are found to cause antibiotic resistance, due to ineffective drug penertration and inability to attain minimal inhibitory concentraton. ${ }^{9}$

Thus, this study will be useful for the medical practitioners in aiding their decision making regarding diagnosis and therapeutics (for active smokers as well as for the pediatric group - as children form one of the most affected groups among passive smokers).

The objectives of this study are:

1. To investigate the effects on respiratory pathogens in relation to biofilm formation following exposure to cigarette smoke.

2. To identify whether the exposure of the respiratory pathogens to CS resulted in dose-related augmentation of biofilm formation.

\section{Materials and Methods}

This prospective study was carried out in the department of Microbiology, Sri Muthukumaran Medical college Hospital and Research Institute, Chennai for a period of 6 months. Before the conduction of the study Institutional ethical committee approval was obtained.

Sample collection and Processing: The respiratory samples received at the tertiary care teaching hospital laboratory from different IPDs and OPDs during the study period were processed; the ones which showed positive for growth were included. So, when the patients came back to the laboratory to receive the report, the nature and the purpose of the study was explained. Informed consent was obtained from those subjects who 
were willing to participate in the study. The participant details were then collected.

Inclusion criteria: only the respiratory isolates of non-smokers were included in the study with participant consent.

\section{Laboratory identification of isolates}

After collection, samples were processed and isolates were identified based on colonial morphology, cultural characteristics and biochemical tests according to standard microbiological laboratory techniques. ${ }^{10}$

Processing of the selected isolates: The isolates were then grown overnight in TSB (tryptic soy broth) to an optical density of 1 at $600 \mathrm{~nm}$. (i.e.; OD of 0.6 to 1.0 ). The overnight cultures were then washed in TSB and re-suspended in TSB at a dilution of 1:40. (Fig. 1)

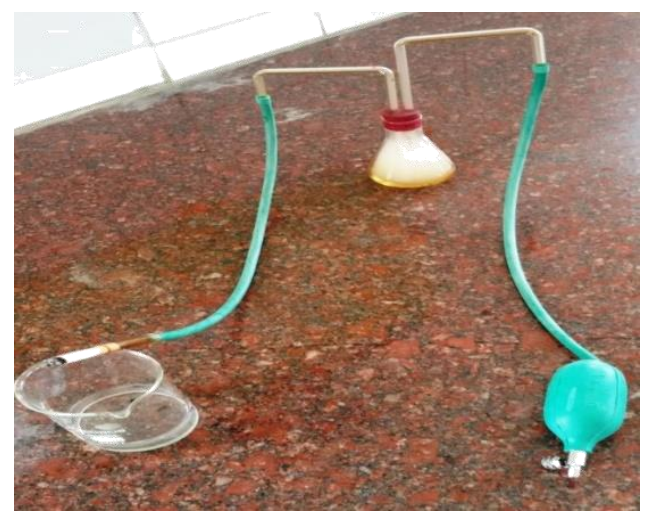

Fig. 1: Preparation of Cigarette Smoke Extract

Preparation of cigarette smoke extract (CSE): Cigarette smoke extract in three concentrations (1cigarette/20ml TSB, 2cigarettes/20ml TSB and 3cigarettes $/ 20 \mathrm{ml}$ TSB) were prepared by bubbling smoke from cigarettes into TSB (Tryptic soy broth)at a rate of one cigarette per 2 min, as described previously $^{(11)}$. Fresh CSE was prepared just before the bacteria are exposed to it (within one hour of experiment). The $\mathrm{pH}$ of the CSE was adjusted to 7.4 and the CSE was sterile filtered through a $0.2 \mathrm{mM}$ filter (Whatman filters).

The CSE preparation was standardized by measuring its absorbance (optical density at $320 \mathrm{~nm}$ ). The spectrographic pattern of absorbance at $320 \mathrm{~nm}$ showed very little variation between different preparations of CSE. (Fig. 2).

Bacteria exposed to cigarette smoke extract (CSE) and Biofilm formation: For every batch tested, $100 \mu 1$ of plain TSB broth was included as negative control (NC).With reference to a single sample, $100 \mu 1$ of the diluted culture was added to 3 wells and then each one of the wells was filled with $100 \mu$ l of CSE of specific concentration. Incubated at $37^{\circ} \mathrm{C}$ overnight. In addition to the three wells, in the fourth well the culture along with plain broth is placed (to identify the bioifilm producer prior to exposure).

After incubation, the cells are dumped out by turning the plate. The biofilms were then washed gently with $0.9 \% \mathrm{NaCl}$ three times. Add $125 \mu \mathrm{l}$ of $0.1 \%$ crystal violet in water to each well. Incubate the microtitre plate for $10-15 \mathrm{mins}$ at room temperature. Then the plate was rinsed 3-4times with water, shake out and blot on a stack of paper towels to remove excess of cells and the stain. $^{16}$

Quantification of the biofilm: (Microtitre Dish Biofilm Formation Assay) $125 \mu \mathrm{L}$ of $30 \%$ acetic acid in water was added to each well to solubilize the crystal violet. The plate was incubated at room temperature for 10-15mins. The absorbance is quantified using a microtitre plate reader at $550 \mathrm{~nm}$ using $30 \%$ acetic acid in water as the blank. ${ }^{12}$

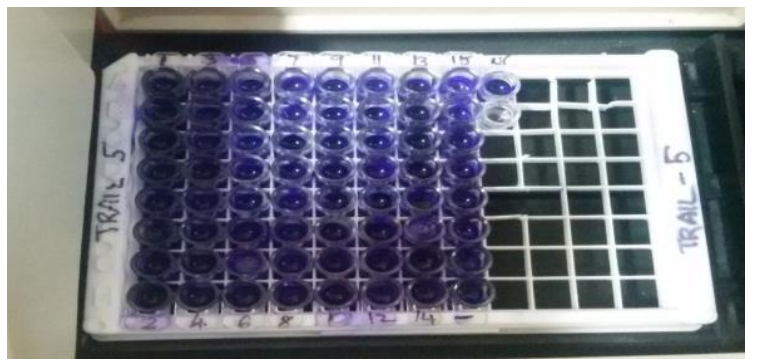

Fig. 2: Microtitre Dish Biofilm Formation Assay

Data recording: Each reading recorded was an average of three recordings. Finally, for each isolate 4 final readings are obtained. The interpretation of biofilm production was done according to the criteria of Stepanovic et al. ${ }^{13}$

Interpretation of OD values: Average OD of the negative control $+3 \times$ standard deviation of negative control was taken as the cut off value ODc.

$2 \times$ ODc of the negative control $\geq$ OD of the isolateNo biofilm production

$4 \times$ ODc of the negative control < OD of the isolate Biofilm producer.

The above criteria are used to identify biofilm and non-biofilm producers in the absence of cigarette smoke extract. Augmentation of biofilm in both categories were further analyzed.

\section{Data analysis}

Fischer's exact test was used to compare the biofilm production status and the change. The two-tailed p-value was less than 0.0001 , considered as extremely statistically significant. Percentages were calculated for discrete variables and confidence interval at $95 \%$ was derived.

\section{Results}

During the study period a total of 77 respiratory samples of non-smokers were analyzed. Out of 77 
clinical isolates $43(55.84 \%)$ were found to be gram positive bacteria and $34(44.16 \%$ ) were found to be gram negative bacteria. Distribution of the species isolated is shown in (Figure 3).It can be seen Staphylococcus aureus $42(54.54 \%)$ being the most common isolate followed by Pseudomonas aeruginosa 18 (23.38\%), Klebsiella pneumonia $8(10.39 \%)$, E.coli $8(10.39 \%)$ and Streptococcus pneumoniae 1 (1.3\%)

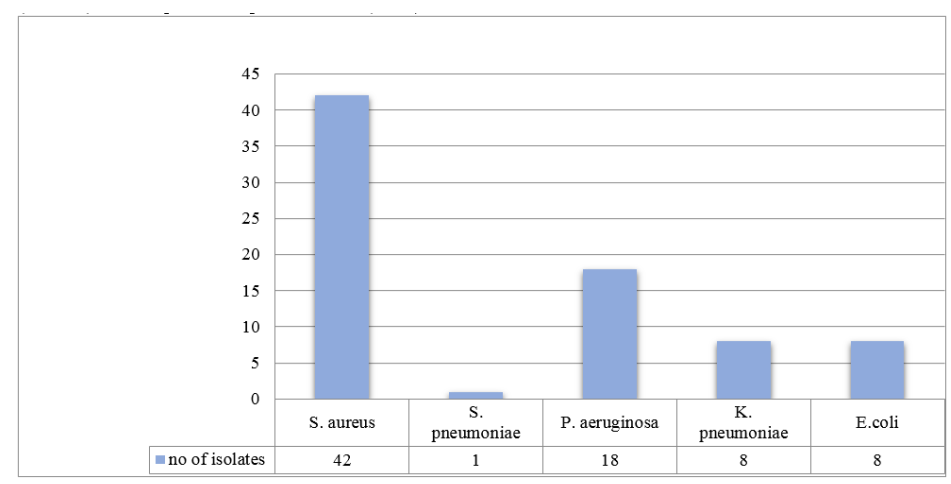

Fig. 3: Bacteriological profile of respiraory isolates

From the total of 77 respiratory isolates, 52(67.53\%) of them had the ability to produce biofilm prior to CSE exposure, the remaining $25(32.47 \%$ ) were non-biofilm producers. It is important in the aspect to note if CSE is able to cause an initiation of biofilm formation in non-biofilm producers.

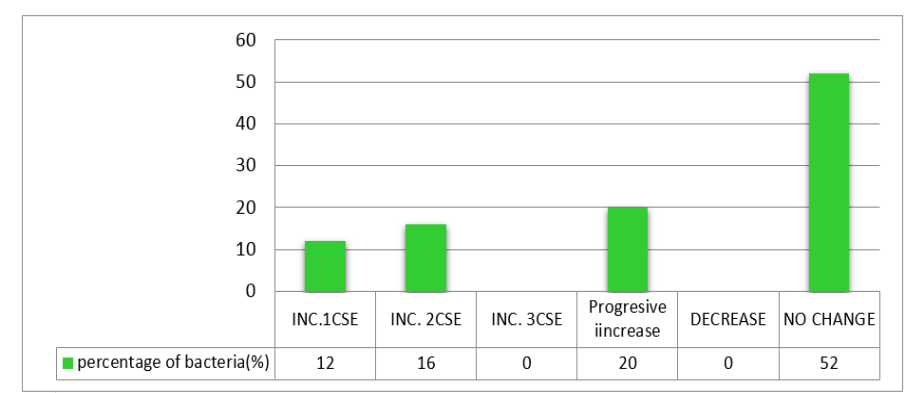

Fig. 4: Impact of CSE on non-biofilm producers

A majority $13(52 \%)$ of the non biofilm producers remained unchanged even after exposure to CSE.5(20\%) of the non-biofilm producers showed progressive increase in biofilm production. About 3(12\%) and 4(16\%) showed an increase with 1CSE and 2CSE respectively. It is essential to note that in a total of 12(48\%) isolates CSE has caused an initiation/induction of biofilm formation in addition to the increase.

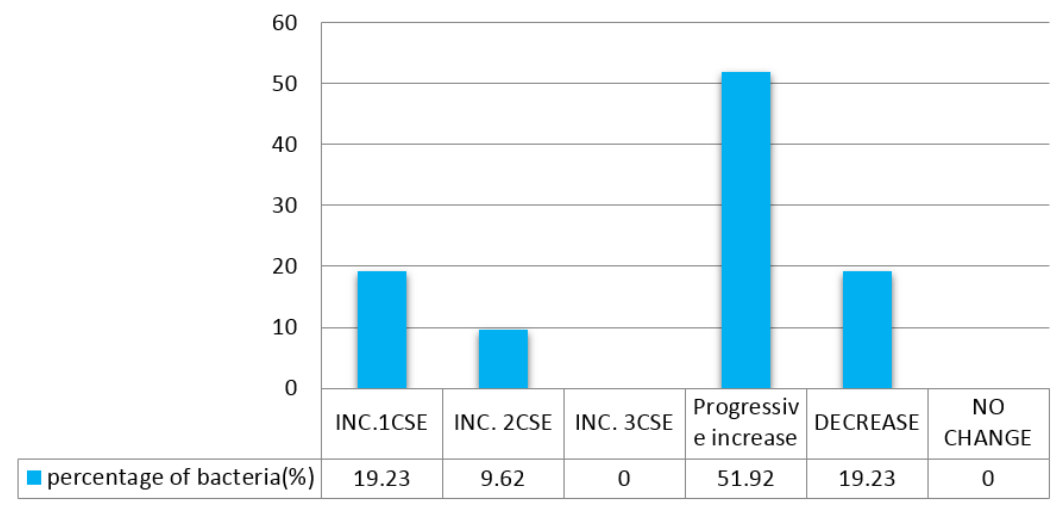

Fig. 5: Impact of CSE on biofilm producers

Among 52 biofilm producers, 27(51.92\%) of the biofilm producers showed progressive increase (dose related augmentation). Following which $10(19.23 \%)$ showed an increase with 1CSE and $5(9.62 \%)$ with 2CSE. It is also observed that $10(19.23 \%)$ showed a decrease in biofilm production.

Among the 77 respiratory isolates included under the study, irrespective of the gram stain status and biofilm formation status, a majority of 32(41.57\%) of showed a dose-related augmentation of biofilm formation on exposure 
to CSE (95\% CI;30.6\% to 52.6\%). In addition to that, $16.88 \%$ (95\% CI;8.5\% to $25.3 \%$ ) and $11.68 \%$ (95\% CI;4.5\% to $18.9 \%$ ) of the isolates showed an increase in biofilm formation with 1CSE and 2CSE respectively. In contrast to the above findings a small portion, $10(12.99 \%)$ of the samples showed a decrease in biofilm formation and $13(16.88 \%)$ isolates remained unchanged even after exposure to CSE. (Table 1)

Table 1: Overall impact of cigarette smoke on the respiratory pathogens

\begin{tabular}{|l|c|}
\hline $\begin{array}{l}\text { Change in Biofilm } \\
\text { Index (Bfi) }\end{array}$ & $\begin{array}{c}\text { Number Of } \\
\text { Organisms n(\%) }\end{array}$ \\
\hline Inc. 1CSE & $13(16.88)$ \\
\hline Inc.2CSE & $9(11.68)$ \\
\hline Inc.3CSE & 0 \\
\hline $\begin{array}{l}\text { Progressive } \\
\text { Increase }\end{array}$ & $32(41.57)$ \\
\hline Decrease & $10(12.99)$ \\
\hline No Change & $13(16.88)$ \\
\hline
\end{tabular}

Therefore, it is notable that a majority of isolates 54(70.13\%) showed an increase in biofilm formation following exposure to CS.

\section{Discussion}

Emergence of multidrug resistant (MDR) respiratory isolates posed a great challenge for the physician in day today practice. This study provides an insight into the relevance of seeking smoking history from the patients to predict the possibility of biofilm producer.

In this study, a total of 77 respiratory isolates of non-smokers were analyzed. Among the respiratory isolates, we observed predominance of Gram positive bacteria over Gram negative bacteria. A similar finding was reported in a recent study in Gujarat by Patel et al., who observed $52 \%$ occurrence. ${ }^{14} 60 \%$ of gram positive respiratory isolates by another similar study. ${ }^{15}$

Staphylococcus aureus was the most predominant organism isolated in our study. In a recent study Sarmah et al.,mentioned Staphylococcus aureus as the predominant gram positive pathogen which is in accordance with our study findings.In contrast to the above few studies reported Streptococcus pneumoniae as predominant pathogen. ${ }^{(14,15)}$. This may be possibly due to difference in prevalence pattern with geographic distribution and selection of samples as we included only non smokers. Among the gram negative organisms, Pseudomonas aeruginosa was the most common isolate which is in accordance with the findings reported by Chawal et al., in his recent study.

Among non-biofilm producers, majority of $13(52 \%)$ of the isolates have remained unchanged on exposure to CSE.It is important to note that in those 12(48\%) of the isolates CSE has not only caused an increase but also an induction/initiation of biofilm formation, as they lacked the ability to produce biofilm prior to CSE exposure. These results are consistent with those observed in the studies by Daphney et $\mathrm{al}^{3}$ Goldstein Daruech et al, ${ }^{6}$ and Cockeran et al, ${ }^{18}$ all of which show that CSE has the ability to both initiate and increase biofilm formation respiratory pathogens.
Among biofilm producers, a majority of $27(51.92 \%)$ of the isolates showed a progressive increase in biofilm formation. Unfortunately other similar studies had not included biofilm producing isolates. To the best of our knowledge, this would be the first study documenting the effect of CSE on biofilm producing isolates.

Progressive increase in biofilm formation is seen in a majority of $32(41.57 \%$ ), which can be attributed to ability of increasing concentrations of the CSE to augment bioifilm formation. This is similar to the observations by Kulkarni et a ${ }^{19}$ based on the research carried out on Staphylococcus aureus. Kolappan et al, ${ }^{20}$ has also observed a similar dose related increase in biofilm formation. It is noted that a total of 54(70.13\%) of the isolates showed an increase in biofilm formation which is consistent with the findings of a research conducted by Goldstein-Daruech et $\mathrm{al}^{(6)}$ who demonstrated that exposure to cigarette smoke increased biofilm formation by $75 \%$. Similar results were also observed by Atunes et $\mathrm{al}^{21} \mathrm{~A}$, who showed that the biofilm mass increases with a increase in exposure to CSE.A research conducted in the University of Louisville by Justin et al also showed that tobacco augments bioiflm in multiple human pathogens. ${ }^{1}$

The following are the limitations of the current study: This is an in vitro technique which may not entirely represent the exposure of the airways to cigarette smoke. This was a short term study, a study of larger cohorts may have been better. Scope of this study does not extend into the molecular level which would be important for the better understanding of the effect of CSE on the change in biofilm index of respiratory pathogens.

\section{Conclusions}

The study has shown that CSE has a significant impact on biofilm formation. Majority of the gram positive and the gram negative bacteria have shown a progressive increase in the biofilm formation on in-vitro exposure to CSE. The ability of CSE to cause 
induction of biofilm is notable among the non biofilm producer. Therefore, smokers have an increased risk of developing respiratory tract infections which may be long standing and difficult to treat. More the amount of cigarette smoke a person is exposed higher may be the risk of developing persistent infections, which are difficult to treat.

\section{Acknowledgement}

The third author (Darshini Venugopal) acknowledges the Indian Council of Medical Research, New Delhi, for providing Short Term Studentship.

\section{References}

1. Justin A Hutcherson, David A Scott, and Juhi Bagaitkar, Scratching the surface - tobacco-induced bacterial biofilms, Tob Induc Dis. 2015; vol. 13(1).

2. Kulkarni R, et al., Cigarette smoke increases Staphylococcus aureus biofilm formation via oxidative stress., Infect Immun , 2012; vol.80(11):380411.

3. N. Daphney Mutepe. Et al, Effects of cigarette smoke condensate on pneumococcal biofilm formation and pneumolysin, Eur Respir J, 2013; vol.(41):392-5.

4. Antunes MB, et al., Molecular basis of tobacco-induced bacterial biofilms. Otolaryngol Head Neck Surg,2012:vol.147(5):876-84.

5. David C. Holzman. Smithsonian cigarette smoke may increase microbial virulence. The Atlantic Monthly, and the Journal of the National Cancer Institute.

6. Goldstein-Daruech. et al, Tobacco Smoke Mediated Induction of Sinonasal Microbial Biofilms, journal.pone., 2011;vol.6(1)

7. Marshall et al, Contributions of Antibiotic Penetration, Oxygen Limitation, and Low Metabolic Activity to Tolerance of Pseudomonas aeruginosa Biofilms to Ciprofloxacin and Tobramycin., Antimicrob Agents Chemother., 2003;Jan;vol.47(1):317-23.

8. Srinivasarao $\mathrm{r}$, correlation between biofilm production and multidrug resistance in imipenam resistant clinical isolates acinetobacter, IJM,2008;2(26):333-7.

9. Philip S Stewart, J William Costerton, Antibiotic resistance of bacteria in biofilms, 2001; 358,(9276):135-

10. Koneman EW, Allen SD, Janda WM, Schreckenberger PC, winn WC. Color Atlas and Textbook of Diagnostic Microbiology. 4th edition. Philadelphia, Pa, USA: JB Lippincott; 1992.

11. Jin Sook Yoon, Hyun Jung Lee1,Min Kyung Chae, Sang Yeul Lee and Eun Jig Lee.Cigarette smoke extract-induced adipogenesis in Graves' orbital fibroblasts is inhibited by quercetin via reduction in oxidative stress.Journal of Endocrinology (2013:) 2(16), 145-56.

12. George A. O' Toole., Microtiter Dish Biofilm Formation Assay J Vis Exp. 2011;(47):2437.

13. Stepanovic S, Vukovi D, Hola V et al. Quantification of biofilm in microtiter plates: overview of testing conditions and practical recommendations for assessment of biofilm production by Staphylococci. APMIS. 2007:1(15):891-9.

14. Patel AK, Luhadia AS, Luhadia SK (2015) Sputum Bacteriology and Antibiotic Sensitivity Pattern of Patients Having Acute Exacerbation of COPD in India - A Preliminary Study. J Pulm Respir Med 2015:5:238.

15. Manikandan, C. and Amsath, A. 2013. Antibiotic susceptibility of bacterial strains isolated from patients with respiratory tract infections. Int. J. Pure Appl. Zool., 2013:1(1):61-9.

16. Sarmah N, Sarmah A, Das DK. A Study on the Microbiological Profile of Respiratory Tract Infection (RTI) in Patients Attending Gauhati Medical College \& Hospital. Ann. Int. Med. Den. Res. 2016; 2(5):MB11-MB15.

17. Chawla K, Mukhopadhay C, Majumdar M, Bairy I. (2008) Bacteriological profile and their antibiogram from cases of acute exacerbations of chronic obstructive pulmonary disease: A hospital based study. Journal of Clinical and Diagnostic Research 2008:612-6.

18. Cockeran et al, Effects of exposure to cigarette smoke condensate on pneumococcal gene expression in relation to biofilm formation, thorax journal, 2012-2026.

19. Kulkarni R, et al., Cigarette smoke increases Staphylococcus aureus biofilm formation via oxidative stress., Infect Immun,2012;vol.80(11):3804-11.

20. C Kolappan, P G Gopi.Tobacco smoking and pulmonary tuberculosis Thorax 2002;57:964-966 31 July 2002

21. Antunes MB, et al., Molecular basis of tobacco-induced bacterial biofilms. Otolaryngol Head Neck Surg, 2012, vol.147(5):876-84. 